\title{
The Expander-Implant Breast Reconstruction in the COVID Era: Which is the "Unhappy" Tissue Expander Priority?
}

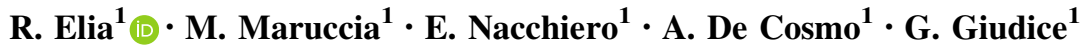

Received: 15 April 2021 / Accepted: 15 April 2021/Published online: 28 April 2021

(C) Springer Science+Business Media, LLC, part of Springer Nature and International Society of Aesthetic Plastic Surgery 2021

\begin{abstract}
Breast surgeons seem to agree on the fact that a same-day surgery (mastectomy and breast reconstruction) protocol provides appropriate cancer treatment during times of unprecedented resource limitations, such as in the COVID era. In this scenario, pre-pectoral implant-based breast reconstruction can be definitively considered a sustainable technique. Nevertheless, the authors focus on the management of patients who had already undergone a same day procedure with two-stage breast reconstruction, implanting a breast tissue expander during the last two-year period and have been progressively delayed according to a surgical care based on priority. We coined the expression "unhappy tissue expander" to define all those symptomatic patients for which surgery should not be delayed even during an epidemic context.

Level of Evidence $V$ This journal requires that authors assign a level of evidence to each article. For a full description of these Evidence-Based Medicine ratings, please refer to the Table of Contents or the online Instructions to Authors www.springer.com/00266.
\end{abstract}

Keywords Tissue expander · COVID · SARS COV2

\section{Dear Sir,}

The capacity to prioritize surgical care delivery remains imperative. In the COVID era, every hospital must develop case-selection protocols based on clinical acuity and

R. Elia

rossellaelia4@gmail.com

1 Division of Plastic and Reconstructive Surgery, Department of Emergency and Organ Transplantation, University of Bari, Piazza Giulio Cesare 11, 70124 Bari, Italy available resources in the context of an effective response strategy [1]. The effort is, indeed, to decrease disease transmission and conserve personal protective equipment.

In this scenario, surgeons have witnessed one of the most dramatic changes in their practices with rapidly decreasing numbers of "elective" surgeries. Interpreting the meaning of "elective" and balancing this definition with the health of the patient can become a challenge for even the most experienced surgeons.

By reference to the definition of priority classes by the Italian State-Regional Agreement for the triennium 2019-2021 [2], the surgeries allowed during the COVID19 outbreak in our Region were those considered "not procrastinating", namely: (1) urgent pathologies; (2) oncological diseases; (3) elective non oncologic disease with high priority class (class A); (4) rare diseases. Conversely, all those elective diseases with lower priority class (B-C-D) were defined as procrastinating and, therefore, postponed [3].

Focusing on breast surgery, surgeons seem to agree on the fact that a same-day surgery (mastectomy and breast reconstruction) protocol provides appropriate cancer treatment during times of unprecedented resource limitations. Previous studies have demonstrated the safety and efficacy of same-day mastectomy and breast reconstruction, largely relying on extensive perioperative planning and postoperative recovery protocols to simplify surgical course and minimize patient morbidity [1]. In relation to the reconstructive procedure, pre-pectoral implant-based breast reconstruction can be definitively considered a sustainable technique. Indeed, in an epidemic context, the plastic surgeon's purpose should be to achieve a satisfactory aesthetic result by adopting the easiest technique, limiting as much as possible operating times, risk of postoperative complication, duration of hospitalization, 
and outpatient visits [4, 5]. Either way, no guidelines on breast reconstructive surgery have been published to date.

After all the overexposed considerations, how to deal with patients who had already undergone a same day procedure with two-stage breast reconstruction, implanting a breast tissue expander during the last 2-year period? We should take into account that so many federal and state orders have been issued to rationalize hospital admissions at various times over the last year, leaving hospital leadership with little guidance on how to interpret the restrictions but ultimately postponing these women's treatment (definite implant reconstruction) as considered a procrastinating surgery.

To develop a framework to help making decisions around not procrastinating surgery, we coined the expression "unhappy tissue expander" to mean all those clinical conditions characterized by chronic pain, shoulder dysfunction, implant malposition, leak or rupture, seroma, and capsular contracture aside from mastectomy flap necrosis, infection and implant exposure that could be considered for urgency.

It is true that, as per the World Health Organization (WHO) statement, our aim and obligation should be "to stop, contain, control, delay and reduce the impact of this virus at every opportunity" [6] but we strongly believe that denying the second stage of the implant-based breast reconstruction in "unhappy patients" as those affected by an "unhappy tissue expander" would generate unfavorable consequences. First, a significant backlog of patients requiring these reconstructive procedures; second, an unacceptable substandard of care and lastly additional patient psychosocial distress.

In conclusion, it is imperative that a hospital should be compliant based on state and national guidelines. However, surgeon leaders need to synthesize national, state and local data to make the best decisions for their patients locally. Focusing back on two-stage breast reconstructions, we consider it reasonable that symptomatic patients with an "unhappy tissue expander" should be eligible for surgery even during an epidemic context.

While the current COVID-19 pandemic appears to be resolving, it is likely that further pandemics, with third and even fourth waves can occur over the coming months and potentially years. Hence, it is imperative that robust protocols are now conceived, to avoid undue delay in decision-making in the future [7].

\section{Declarations}

Conflict of interest All authors hereby declare not to have any potential conflict of interests and not to have received funding for this work. Each author participated sufficiently in the work to take public responsibility for the content and agree to its publication.

Human Participants or Animals This article does not contain any studies with human participants or animals performed by any of the authors.

Informed Consent For this type of study informed consent is not required.

\section{References}

1. Specht M, Sobti N, Rosado N et al (2020) High-efficiency sameday approach to breast reconstruction during the COVID-19 crisis. Breast Cancer Res Treat. 182(3):679-688. https://doi.org/10.1007/ s10549-020-05739-7

2. http://www.salute.gov.it/portale/listeAttesa/ dettaglioPubblicazioniListeAttesa.jsp?lingua $=$ italiano\&id $=2824$

3. Elia R, Giudice G, Maruccia M (2021) Corrigendum to "Plastic Surgery in the time of Coronavirus in Italy Can we really say 'Thanks God we are plastic surgeons?'” [Journal of Plastic, Reconstructive \& Aesthetic Surgery, Vol. 73(11) 2020, 2086-2102]. J Plast Reconstr Aesthetic Surg JPRAS 74(1):245.

4. Salgarello M, Adesi LB, Visconti G, Pagliara DM, Mangialardi ML (2020) Considerations for performing immediate breast reconstruction during the COVID-19 pandemic. Breast J. https:// doi.org/10.1111/tbj.13876

5. Maruccia M, Elia R, Gurrado A et al (2020) Skin-reducing mastectomy and pre-pectoral breast reconstruction in large ptotic breasts. Aesthetic Plast Surg. https://doi.org/10.1007/s00266-02001616-2

6. Coles CE, Aristei C, Bliss J et al (2020) International Guidelines on radiation therapy for breast cancer during the COVID-19 pandemic. Clin Oncol R Coll Radiol G B 32(5):279-281. https:// doi.org/10.1016/j.clon.2020.03.006

7. Jallali N, Hunter JE, Henry FP et al (2020) The feasibility and safety of immediate breast reconstruction in the COVID-19 era. J Plast Reconstr Aesthet Surg 73(11):1917-1923. https://doi.org/ 10.1016/j.bjps.2020.08.044

Publisher's Note Springer Nature remains neutral with regard to jurisdictional claims in published maps and institutional affiliations. 